was used to identify factors that increased length of stay beyond the median for the overall population.

Results We identified 719 exacerbations between 1/4/2013- 31/ $3 / 2014$. The number of exacerbations ranged from 157-228/ quarter with the winter quarters (Q3/4) being associated with the highest exacerbation frequency. Exacerbators had a median (IQR) age of 46 (31-64) years, length of stay of 2 (1-5) days, Charlson score 4[4-4]. The majority of patients were female (72\%). The percentage of repeat attender was $12 \%$ of the total population with the highest percentage in the winter quarters. Logistic regression models identified that ethnicity (non-white/ Caucasian), baseline admission CRP, neutrophil count, age, coding of status asthmaticus and Charlson co morbidity index significantly increased length of stay beyond the median of 2 days. In contrast neither admission or highest ever blood eosinophil count influenced the median length of stay.

Conclusions We have identified a number of factors associated with an increased length of stay in asthma hospital exacerbations in Leicester. Our observations support the notion that age, comorbidity and biochemical features of infection rather than eosinophilic inflammation increase length of stay. Further research is required to examine the mechanisms that underpin asthma admissions in this population and to reduce length of stay.

\section{COPD phenotyping}

\section{P58 A COMPARISON BETWEEN THE CLINICAL FEATURES OF PISZ AND PIZZ PATIENTS WITH ALPHA-1 ANTITRYPSIN DEFICIENCY}

'S Vayalapra, ${ }^{2} \mathrm{RG}$ Edgar, ${ }^{2} \mathrm{D}$ Griffiths, ${ }^{2} \mathrm{RA}$ Stockley, ${ }^{1} \mathrm{AM}$ Turner. 'University of Birmingham, Birmingham, UK; ${ }^{2}$ University Hospitals Birmingham NHSFT, Birmingham, UK

\subsection{6/thoraxjn-2014-206260.199}

Introduction Alpha-1 antitrypsin deficiency (AATD) is a hereditary disorder affecting about 1 in 3000 people in the UK commonly associated with early-onset emphysema. There are two common deficiency alleles - PiS and PiZ. PiZZ patients have severe AATD, with levels of $10-15 \%$ normal. PiSZ patients have less severe deficiency $(\approx 40 \%$ normal) and are generally thought to have a minimal risk. We hypothesised that if PiSZ patients were at lower risk of COPD than PiZZ, and their lung disease would be more characteristic of usual COPD than that of PiZZ patients.

Method 104 PiSZ patients and 638 PiZZ patients from the UK AATD registry (ADAPT) were compared for their demographics, lung function, risk factors for COPD (e.g. smoking, occupation), co-morbidities associated with COPD, index status (i.e. if diagnosed due to lung disease or family screening) and CT densitometry (where available). Outcome in terms of lung function decline and mortality was also assessed. Univariate statistics were used to guide subsequent regression analyses.

Results Emphysema was more likely in PiZZ than PiSZ patients (OR 11.0 (5.7-21.3); p < 0.001) in the regression analysis after accounting for age, pack years and lung index status. PiZZ patients also had significantly worse FEV1 and DLCO than PiSZ patients in similar regression models (both $\mathrm{p}<0.01$ ). Emphysema was more severe in both upper and lower zone (both $p<$ 0.01 ), and proportionately greater in the lower zone (UZ/LZ VI
$=1.5 \mathrm{v} 1.2)$ in PiZZ patients. Mortality and DLCO decline were also greater in PiZZ patients.

Conclusion PiSZ patients have a milder form of AATD associated with better lung function. The data suggests the pattern of emphysema is closer to usual COPD than classical AATD. Further analyses comparing PiSZ to PiMM are now ongoing.

\section{P59 UTILITY OF FIB4 SCORE AND LIVER DISEASE IN ALPHA- 1 ANTITRYPSIN DEFICIENCY (A1ATD)}

'D Bruce-Hickman, 'AD Saleh, 'B Gooptu, 'DA Lomas, ${ }^{2} D$ Thorburn, 'JR Hurst. 'The London Alpha-1 Antitypsin Deficiency Service, UCL Respiratory, London, UK; ${ }^{2}$ Department of Hepatology, Royal Free London NHS Foundation Trust, London, UK

\subsection{6/thoraxjn-2014-206260.200}

Introduction A1ATD is an autosomal co-dominant condition where homozygosity for the Z-allele results, classically, in emphysematous lung involvement, and liver fibrosis dependant on polymerisation rate of aberrant protein. ${ }^{1}$ The FIB4 score non-invasively estimates risk of liver fibrosis, ${ }^{2}$ but has not previously been investigated in A1ATD. We completed preliminary assessment of the utility of FIB4 in our A1ATD cohort. Whilst the standard of care in A1ATD should be joint respiratory-hepatology services, not all patients are able to access this. A simple tool to guide referral to hepatology services could therefore be clinically useful to the respiratory community.

Methods We report data from 30 PiZZ patients with ultrasound (USS) characterisation of liver disease. An abnormal USS was considered as any abnormality other than cysts, thus including features of cirrhosis and fatty infiltration. FIB4 was calculated as [Age (years)xAST(U/l)]/[Platelets $\left.\left(10^{9}\right) x A L T(U / 1)\right]$. The most recent lung physiology was recorded as FEV1 (\%predicted), diffusion coefficient (KCO,\%predicted) and residual volume (RV,\% predicted). Body mass index (BMI) was calculated.

Results The 30 patients had a mean age of $54 \pm 12.4$ years, 14 were male. Lung function showed mean FEV1 $1.85 \pm 1.12 \mathrm{~L}$

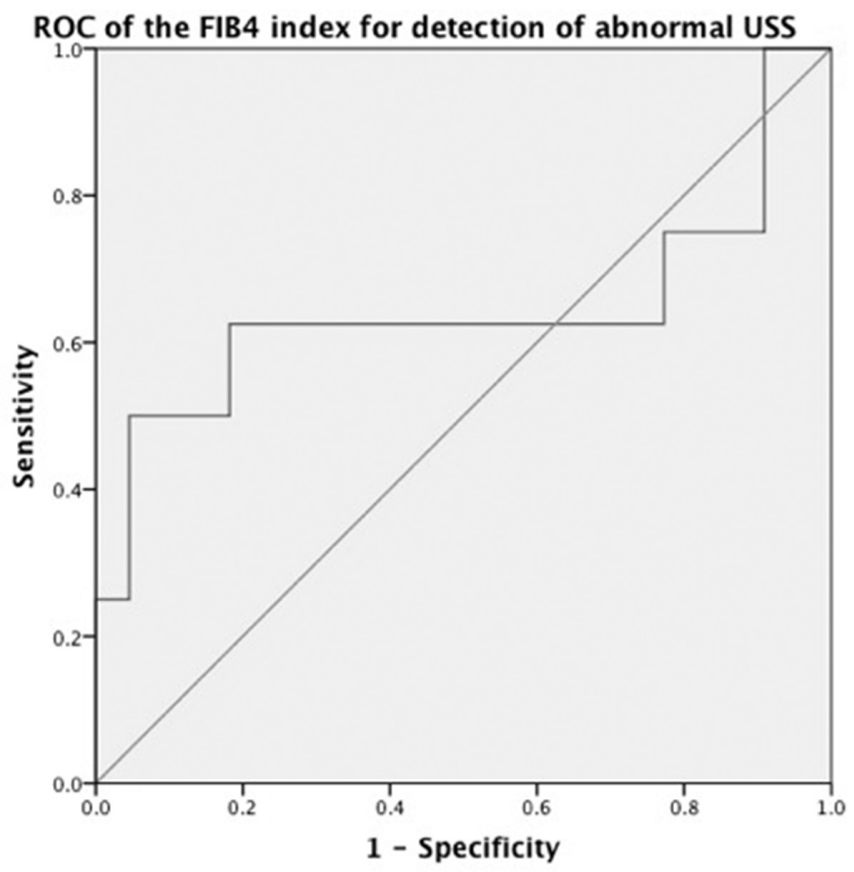

Abstract P59 Figure 1 ROC of the FIB4 index for detection of abnormal USS 